\title{
$k$-Nearest Neighbor Classification on First-Order Logic Descriptions
}

\author{
S. Ferilli \\ M. Biba \\ T.M.A. Basile \\ N. Di Mauro \\ F. Esposito \\ Dipartimento di Informatica \\ Università di Bari \\ via E. Orabona, 4 - 70125 Bari - Italia \\ \{ferilli, biba, basile, ndm, esposito\}@di.uniba.it
}

\begin{abstract}
Classical attribute-value descriptions induce a multidimensional geometric space on which computing distance between descriptions reduces to evaluating an Euclidean distance between tuples of coordinates. This is the base on which a large part of the Machine Learning literature has built its methods and techniques. Unfortunately, when First-Order Logic descriptions must be taken into account to deal with domain complexity, the problems of non-fixed descriptions and indeterminacy make computation of the distance between descriptions much less straightfoward and hence prevent the use of distance-based techniques. This paper proposes the exploitation of a novel framework for computing the similarity between relational descriptions in a classical instance-based learning technique, $k$-Nearest Neighbor classification. Experimental results on real-world datasets show good performance, comparable to that of state-of-the-art conceptual learning systems, which supports the validity of the proposal.
\end{abstract}

\section{Introduction}

Classical attribute-value descriptions of objects gained wide acceptance and success in the Machine Learning community because, being composed of a fixed number of features for each of which a specific value is provided, the attributes can be seen as dimensions in a multi-dimensional geometric space, and the related values as corresponding coordinates, so that each possible described object can be univoquely mapped onto, and identified by, a single point of the space. This representation allowed the development of grouping and discrimination techniques that cast learning as an application based on mathematical and geometrical concepts and properties. The core facility of associating objects to points in a geometric space resides in the possibility of straightforwardly assessing how much two given objects are similar to, or different from, each other as a simple application of the Euclidean distance between the corresponding coordinate vectors (symbolic features can be easily mapped onto discrete numerical coordinates, as well). This allowed for high performance of the techniques, but finds its limit in the rigidity of the descriptions that must identify a fixed number of features in which capturing all possible situations, and that must include all (and, possibly, only) those that are significant for the given task.

However, the complexity of some domains cannot be captured by simple attribute-value descriptions, but requires the possibility of including a variable number of objects and features in the descriptions and the ability to express relations between objects. To deal with such domains, First-Order Logic ( $F O L$ for short) represents a suitable formalism that can overcome the typical limitations shown by propositional or attribute-value representations. As a consequence and tradeoff for its expressive power, however, there is no more a fixed way for comparing two descriptions, but various portions of one description can be possibly mapped in (often many) different ways onto another description. This problem, known as indeterminacy, not only causes a significant computational effort when two descriptions have to be compared to each other, but also excludes a straightforward computation of the distance between them, since FOL does not induce anymore a Euclidean space in which reusing consolidated mathematical and geometrical notions. This explains why much less work has been done in the Machine Learning literature on distance-based methods and techniques for FOL descriptions than for propositional ones.

This paper aims at contributing in this critical area, proposing the adoption of a novel framework, that supports the comparison between FOL formulæ, to per- 
form similarity-based Machine Learning in relational domains. This allows many applications, covering both unsupervised and unsupervised learning, and ranging from (conceptual) Clustering to Instance-based techniques. In particular, it focusses on $k$-Nearest Neighbor ( $k$-NN for short), that strongly rely on the availability and quality of similarity measures for classifying unseen observations according to the closest known prototypes. In addition to yielding a similarity evaluation of entire descriptions, the proposed framework allows to compare also description components, suggesting those that are more similar and hence more likely to correspond to each other and this way tackling indeterminacy. Since this concerns the semantic aspects of the domain, and hence there is no precise (i.e., algorithmic) way for recognizing the correct (sub-)formulæ to be associated, the problem is attacked based only on syntactic structure.

In the following sections, after presenting preliminary notions about the formalism and a brief recall of related work, the similarity framework will be introduced, from the parameters and corresponding formula on which the framework is based, through similarity criteria for descriptions sub-components up to the assessment of similarity between whole clauses. Then, a report of the experiments on $k$-Nearest Neighbor classification in a real-world domain will be provided, before concluding the paper and outlining future work directions.

\section{Preliminaries and Related Work}

Logic Programming [10] is the fragment of FOL that restricts to formulæ in the form of clauses. It is an important paradigm in Artificial Intelligence, and many first-order Machine Learning systems infer theories in the form of logic programs. Logic programs (or theories) are made up of Horn clauses, i.e. logical formulæ of the form $\quad l_{0} \vee \neg l_{1} \vee \cdots \vee \neg l_{n} \quad$ which is equivalent to $l_{1} \wedge \cdots \wedge l_{n} \Rightarrow l_{0} \quad$ usually represented in Prolog style as $l_{0}:-l_{1}, \ldots, l_{n}$ to be interpreted as " $l_{0}$ (called head of the clause) is true, provided that $l_{1}$ and ... and $l_{n}$ (called body of the clause) are all true". The $l_{i}$ 's are atoms (i.e., predicates applied to a number of terms equal to their arity); a literal is an atom (called positive literal) or its negation (called negative literal). Two literals are linked iff they share at least one of their arguments; a clause is linked iff any couple of its literals can be connected by a chain of couple-wise linked literals in the clause. A clause is range restricted iff all terms appearing in the head also appear in the body. Datalog is a restriction of Prolog where only variables and constants are allowed as terms (i.e., it is syntactically the function-free version of Prolog) [2].

Previous work on $k$-NN in FOL includes RIBL [4], a $k$-NN classifier based on a modified version of the similarity function proposed in [1] for the system $K G B$. The basic idea of the measure used in $R I B L$ is that objects are described by values (e.g., size and position) and their relation to other objects. The similarity between two objects is computed by considering the immediate neighborhood of the objects. For instance, the similarity between two identifiers is determined by the similarity between the set of facts where these identifiers occur. Also, the similarity between two facts is determined by the similarity between their arguments. If some of these arguments are in turn identifiers themselves, one can get a loop: therefore, a depth parameter is introduced and similarity is only computed up to this depth. Thus, the similarity among objects depends on the similarity of their attributes' values (e.g., the similarity of their size) and the similarity of the objects related to them. The similarity of the related objects in turn depends on the attribute values of these objects and their relation to other objects and so on. Such a propagation poses the problem of indeterminacy in associations, that our technique avoids thanks to the different structural approach. Although the indeterminacy problem could be handled by using some CSP technique (e.g., [12]), our proposal is exploiting the information provided by the peculiar clause structure. RISE [3] is another system that combines a rule classifier with a $k$-NN technique: when the instance to classify is not covered by any rule, the distance of the instance to the different rules is evaluated and the instance is classified according to the majority vote of its "neighbor" rules .

We will deal with the case of linked Datalog clauses, without loss of generality: indeed, linked sub-parts of non-linked clauses can be dealt with separately (because, having no connection between each other, do not contribute any information that is relevant to describe the head), while the flattening/unflattening procedures [11] can translate generic first-order clauses (allowing also functions as terms) into Datalog ones and viceversa. Moreover, we will assume that examples are represented, according to direct relevance, as ground (variable-free) clauses where the argument(s) of the head represent the (tuple of) object(s) to be classified, the head predicate their class, and the body represents the set of all and only those known literals in the knowledge base that are significant for describing the head, where a literal is relevant if it is (directly or indirectly) linked to the head. Again this is not limiting, since given a general knowledge base it is possible to collect all and only those facts that fulfill such requirement. 
Observations to be classified will be described in the same way, but with a dummy predicate in the head.

Given two Datalog (sub-)formulæ $C^{\prime}$ and $C^{\prime \prime}$, a term association on them is a set of couples of terms, usually written $t^{\prime} / t^{\prime \prime}$, where the $t^{\prime} \in \operatorname{terms}\left(C^{\prime}\right)$ and $t^{\prime \prime} \in \operatorname{terms}\left(C^{\prime \prime}\right)(\operatorname{terms}(\cdot)$ denotes the set of terms appearing in $\cdot)$. In the following, we will call compatible two FOL (sub-)formulæ that can be mapped onto each other without yielding inconsistent term associations (i.e., a term in one formula cannot be mapped onto different terms in the other formula).

\section{The Similarity Framework}

The similarity framework for Horn Clauses on which the work in this paper is based includes a similarity function, but its novelty relies on a set of criteria that focus on particular clause components to tackle the problem of indeterminacy while still preserving a considerable amount of information about the description structures. It is syntax-based, and hence totally general, since it does not assume domain-related knowledge for assessing the similarity degree between two descriptions. Here, we briefly recall it from [8].

Like in classical and state-of-the-art distance measures in the current literature, mostly developed in the propositional setting, are based on these parameters (e.g., those by Tverski, Dice or Jaccard), the evaluation of similarity between two items $i^{\prime}$ and $i^{\prime \prime}$ is based both on the number of common and different features between them [9]:

$n$, number of features owned by $i^{\prime}$ but not by $i^{\prime \prime}$;

$l$, number of features owned both by $i^{\prime}$ and by $i^{\prime \prime}$;

$m$, number of features owned by $i^{\prime \prime}$ but not by $i^{\prime}$.

However, the new formula overcomes the undesirable behaviour of those measures in cases in which $n, l$ or $m$ are 0 ; expressed in terms of the items to be compared or, equivalently, in terms of the corresponding parameters, it is the following:

$\operatorname{sf}\left(i^{\prime}, i^{\prime \prime}\right)=\operatorname{sf}(n, l, m)=\frac{l+1}{2}\left(\frac{1}{l+n+2}+\frac{1}{l+m+2}\right)$

It takes values ranging in the classical spectrum ]0,1[, which can be interpreted as the level of likelihood/confidence that the two items under comparison are actually similar. A complete overlapping of the two $(n=m=0)$ tends to the limit of 1 as long as the number of common features grows, whereas in case of no overlapping $(l=0)$ the function will tend to 0 as long as the number of non-shared features grows. The lefthand-side ratio in parentheses refers to item $i^{\prime}$, while the right-hand-side ratio refers to item $i^{\prime \prime}$, which allows to weight them differently if needed (e.g., when comparing a model to an observation). Using equal weight $(1 / 2)$, as in (1), the function is symmetric with respect to the two items to be compared.

The framework proposed here for Instance-based learning exploits repeatedly and pervasively the above formula in various combinations that assign a similarity degree to progressively complex clause constituents, from terms to atoms to groups of atoms to whole clauses. The similarity of each component type is based on the similarity of simpler components (only), so that no recursion nor indeterminacy can be present. Empirically, one can note that no single component type is by itself neatly discriminant, but their cooperation succeeds in assigning sensible and useful similarity values to the various kinds of components, and in distributing on each kind of component a proper portion of the overall similarity, so that the difference becomes ever clearer as long as they are composed one ontop the previous ones. This makes the proposed approach robust to lacks of information due to some of the components.

In FOL formulæ, terms represent specific objects, that are related to each other by means of predicates. Hence, two main levels of similarity can be defined for pairs of first-order descriptions: the object level, concerning similarities between the objects referred to in the descriptions (and represented by terms), and the structure one, referring to how the nets of relationships in the descriptions overlap (expressed by $n$-ary predicates applied to terms).

\subsection{Object-level Similarity}

Consider two clauses $C^{\prime}$ and $C^{\prime \prime}$. Call $A^{\prime}=$ $\left\{a_{1}^{\prime}, \ldots, a_{n}^{\prime}\right\}$ the set of terms in $C^{\prime}$, and $A^{\prime \prime}=$ $\left\{a_{1}^{\prime \prime}, \ldots, a_{m}^{\prime \prime}\right\}$ the set of terms in $C^{\prime \prime}$. When comparing a pair of objects $\left(a^{\prime}, a^{\prime \prime}\right) \in A^{\prime} \times A^{\prime \prime}$, a first kind of features to be compared is the set of properties they own (characteristic features), usually expressed by unary predicates. For instance, characteristic features for a term representing a person could be young $(X)$ or male $(X)$. A corresponding similarity value, called characteristic similarity, is obtained by applying (1) to the sets $P^{\prime}$ of characteristic features related to $a^{\prime}$ and $P^{\prime \prime}$ of characteristic features related to $a^{\prime \prime}$ :

$$
\operatorname{sf}_{c}\left(a^{\prime}, a^{\prime \prime}\right)=\operatorname{sf}\left(\left|P^{\prime} \backslash P^{\prime \prime}\right|,\left|P^{\prime} \cap P^{\prime \prime}\right|,\left|P^{\prime \prime} \backslash P^{\prime}\right|\right)
$$

Also the ways in which terms relate to each other, generally expressed by the position the term holds among the $n$-ary predicate arguments, determine additional features useful for comparison (relational features): indeed, different positions actually refer to different roles played by the objects. For instance, rela- 
tional features in the predicate parent $(\mathrm{X}, \mathrm{Y})$ are represented by the roles of the parent (first argument position) and of the child (second argument position). Thus, another similarity value, called relational similarity, is based on how many times the two objects play the same or different roles in the $n$-ary predicates, by applying (1) to the multisets $R^{\prime}$ of roles played by $a^{\prime}$ and $R^{\prime \prime}$ of roles played by $a^{\prime \prime}$ (they are multisets because a term can play the same role in different instances of the same predicate):

$$
\operatorname{sf}_{r}\left(a^{\prime}, a^{\prime \prime}\right)=\operatorname{sf}\left(\left|R^{\prime} \backslash R^{\prime \prime}\right|,\left|R^{\prime} \cap R^{\prime \prime}\right|,\left|R^{\prime \prime} \backslash R^{\prime}\right|\right)
$$

These values can be combined, so that the overall object similarity between $a^{\prime}$ and $a^{\prime \prime}$ is defined as

$$
\operatorname{sf}_{o}\left(a^{\prime}, a^{\prime \prime}\right)=\operatorname{sf}_{c}\left(a^{\prime}, a^{\prime \prime}\right)+\operatorname{sf}_{r}\left(a^{\prime}, a^{\prime \prime}\right)
$$

\subsection{Structure-level Similarity}

While comparison among terms still belongs (can be reduced to) the propositional (attribute-value) setting, checking the structural similarity of two formulæ involves the way in which terms are related by means of atoms built on $n$-ary predicates. Hence, it is peculiar to the first-order logic setting, and introduces the problem of indeterminacy in mapping (parts of) a formula into (parts of) another one. This can be reduced to the computation of (sub-)graph homomorphisms, a problem known to be $N P$-hard due to the possibility of mapping a (sub-)graph onto another in many different ways. The proposed focusses on linkedness (i.e., the fact that two atoms share at least one of their arguments) as a feature on which basing the structural similarity assessment.

The simplest relational components in a first-order logic formula are atoms. Thus, a first problem is computing the degree of similarity between two atoms $l^{\prime}$ and $l^{\prime \prime}$. In this case, linkedness can be exploited 'inbreadth', considering the concept of star of an $n$-ary atom (the multiset of $n$-ary predicates corresponding to the atoms linked to it by some common term - indeed, a predicate can appear in multiple instantiations among these atoms). The star similarity between two compatible $n$-ary atoms $l^{\prime}$ and $l^{\prime \prime}$ having stars $S^{\prime}$ and $S^{\prime \prime}$, respectively, can be computed by applying (1) to the number of common and different elements in each of the two stars. However, also the similarity of the objects involved in the two atoms $l^{\prime}$ and $l^{\prime \prime}$ must be taken into account, and hence the star similarity also considers the object similarity for all pairs of terms included in the association $\theta$ that maps $l^{\prime}$ onto $l^{\prime \prime}$ :

$$
\begin{aligned}
\operatorname{sf}_{s}\left(l^{\prime}, l^{\prime \prime}\right)= & \operatorname{sf}\left(\left|S^{\prime} \backslash S^{\prime \prime}\right|,\left|S^{\prime} \cap S^{\prime \prime}\right|,\left|S^{\prime \prime} \backslash S^{\prime}\right|\right)+ \\
& +\operatorname{avg}\left(\left\{\operatorname{sf}_{o}\left(t^{\prime}, t^{\prime \prime}\right)\right\}_{t^{\prime} / t^{\prime \prime} \in \theta}\right)
\end{aligned}
$$

Being able to compare two atoms, the next step is comparing sets of atoms. In this case linkedness can be exploited 'in-depth', considering chains of atoms where each atom is linked to both the previous and the next ones in the chain, but the previous and the next one do not share any argument at all. Such chains, for a clause $C$, can be determined as all possible paths starting from the root and reaching leaf nodes (those with no outcoming edges) in the graph $G_{C}=(V, E)$ built as follows:

- $V=\left\{l_{0}\right\} \cup\left\{l_{i} \mid i \in\{1, \ldots, n\}, l_{i}\right.$ built on $k$-ary predicate, $k>1\}$ and

- $E \subseteq\left\{\left(a_{1}, a_{2}\right) \in V \times V \mid \operatorname{terms}\left(a_{1}\right) \cap \operatorname{terms}\left(a_{2}\right) \neq\right.$ $\emptyset\}$ where the edges are chosen in such a way to obtain a stratified graph in which the head is the only node at level 0 , and each successive level is made up by nodes not belonging to previous levels that have at least one term in common with nodes in the previous level.

When comparing two clauses, their heads (roots of the graphs) are unique (being the only head atom in the clause).

Given two clauses $C^{\prime}$ and $C^{\prime \prime}$ with associated graphs $G_{C^{\prime}}$ and $G_{C^{\prime \prime}}$ respectively, and two paths $p^{\prime}=<$ $l_{0}^{\prime}, l_{1}^{\prime}, \ldots, l_{n^{\prime}}^{\prime}>$ in $G_{C^{\prime}}$ and $p^{\prime \prime}=<l_{0}^{\prime \prime}, l_{1}^{\prime \prime}, \ldots, l_{n^{\prime \prime}}^{\prime \prime}>$ in $G_{C^{\prime \prime}}$, the intersection between $p^{\prime}$ and $p^{\prime \prime}$ is defined as the pair of longest compatible initial subsequences $\left(<l_{1}^{\prime}, \ldots, l_{k}^{\prime}>,<l_{1}^{\prime \prime}, \ldots, l_{k}^{\prime \prime}>\right)$ of $p^{\prime}$ and $p^{\prime \prime}$, excluding the head. Their differences are then defined as the incompatible trailing parts:

$$
\begin{aligned}
& p^{\prime} \backslash p^{\prime \prime}=<l_{k+1}^{\prime}, \ldots, l_{n^{\prime}}^{\prime}>\quad\left|p^{\prime} \backslash p^{\prime \prime}\right|=n^{\prime}-k \\
& p^{\prime \prime} \backslash p^{\prime}=<l_{k+1}^{\prime \prime}, \ldots, l_{n^{\prime \prime}}^{\prime \prime}>\quad\left|p^{\prime \prime} \backslash p^{\prime}\right|=n^{\prime \prime}-k
\end{aligned}
$$

Hence, the path similarity between $p^{\prime}$ and $p^{\prime \prime}$ is computed by applying (1) to the number of atoms in the maximal compatible subsequences and in the trailing parts:

$$
\begin{aligned}
\operatorname{sf}_{p}\left(p^{\prime}, p^{\prime \prime}\right)= & \operatorname{sf}\left(n^{\prime}-k, k, n^{\prime \prime}-k\right)+ \\
& +\operatorname{avg}\left(\left\{\operatorname{sf}_{s}\left(l_{i}^{\prime}, l_{i}^{\prime \prime}\right)\right\}_{i=1, \ldots, k}\right)
\end{aligned}
$$

\subsection{Clause Similarity}

As a final step, consider the case of two clauses $C^{\prime}$ and $C^{\prime \prime}$, whose heads are built on predicates having the same arity. Their bodies can be interpreted as the observations describing the tuple of terms in the head by means of all known facts related to them. Thus, assessing the similarity among those tuples consists in assessing the similarity between the respective bodies. According to the technique presented so far, we need a way to state the number of common and different atoms 
in the two bodies. The set of common atoms can be considered as their least general generalization, i.e. the most specific model for the given pair of descriptions $C=\left\{l_{1}, \ldots, l_{k}\right\}$ for which there exist two substitutions $\theta^{\prime}$ and $\theta^{\prime \prime}$ such that $\forall i=1, \ldots, k: l_{i} \theta^{\prime}=l_{i}^{\prime} \in C^{\prime}$ and $l_{i} \theta^{\prime \prime}=l_{i}^{\prime \prime} \in C^{\prime \prime}$, respectively.

Using the classical $\theta$-subsumption generalization model such a generalization is unique and can be computed according to Plotkin's algorithm, but gives some undesirable side-effects concerning the need of computing its reduced equivalent (and also of some counterintuitive aspects of the result). For this reason, most ILP learners require the generalization to be a subset of the clauses to be generalized, in which case the $\theta_{O I}$ subsumption generalization model [6], based on the Object Identity assumption, represents a supporting framework with solid theoretical foundations to be exploited. The work in [7] shows that the similarity techniques shown so far are also able to guide the generalization procedure in obtaining quickly an accurate approximation of the least general generalization. Un$\operatorname{der} \theta_{O I}$ subsumption, the least general generalization is not unique, but each minimal generalization is already reduced, and hence all of the atoms that make it up are mapped onto different atoms in the generalized clauses, which reduces computation of the common atoms to counting the length of the generalization, and computation of the different ones to subtracting the length of the generalization from that of either clause. The overall clause similarity is computed according to the amount of overlapping and different atoms and terms, taking into account also the star similarity values for all pairs of atoms associated by the least general generalization (which includes both structural and object similarity):

$$
\begin{aligned}
\operatorname{fs}\left(C^{\prime}, C^{\prime \prime}\right)= & \operatorname{sf}\left(\left|C^{\prime}\right|-|C|,|C|,\left|C^{\prime \prime}\right|-|C|\right) . \\
& \cdot \operatorname{sf}\left(n_{o}, l_{o}, m_{o}\right)+ \\
& +\operatorname{avg}\left(\left\{\operatorname{sf}_{s}\left(l_{i}^{\prime}, l_{i}^{\prime \prime}\right)\right\}_{i=1, \ldots, k}\right)
\end{aligned}
$$

where

$n_{o}=\left|\operatorname{terms}\left(C^{\prime}\right)\right|-|\operatorname{terms}(C)|$;

$l_{o}=|\operatorname{terms}(C)|$

$m_{o}=\left|\operatorname{terms}\left(C^{\prime \prime}\right)\right|-|\operatorname{terms}(C)|$.

The first two components are multiplied in order to have a limited influence on the overall similarity, that must be predominantly determined by the similarity of the single atoms.

As to the computational complexity, if $n$ is the number of literals in the longest clause and $m$ is the number of nodes at each level of the clause graph, in the worst case of the atoms being absolutely equally distributed both $i n$ and among levels we have $m=\sqrt{n}$ and hence an order of $\sqrt{n}^{\sqrt{n}}$. However, in more realistic cases, in which atoms are irregularly distributed in breadth and/or depth, and adjacent levels are not completely connected, the complexity moves towards the two extremes $m=1 \Rightarrow m^{n / m}=1^{n}=1$ and $m=n \Rightarrow m^{n / m}=n^{1}=n$.

\section{Experiments}

Some experiments were designed to check whether the proposed framework is actually able to give significant similarity hints when comparing two structures, and hence can represent a good way for supporting $k$ Nearest Neighbor classification on FOL descriptions. All of them were run under WindowsXP Professional on a PC endowed with a $2.13 \mathrm{GHz}$ Intel Dual Core Duo processor and 2GB RAM. 10-fold cross validation was used to test the learning effectiveness. The 10 folds were created so that the distribution of examples from the various classes was uniform in each fold, and consequently each of the resulting 10 training and test set contained approximately $90 \%$ and $10 \%$, respectively, of examples from each class.

A real-world domain that requires first-order logic descriptions for capturing the complexity of the observations was choosen for performing the experiments and assess the applicability and performance of the proposed approach. Specifically, it concerns the descriptions (automatically generated from electronic versions of the documents) of scientific papers layout, according to which identifying the papers' type and significant components ${ }^{1}$. It is made up of 353 descriptions of scientific papers first page layout, belonging to 4 different classes: Elsevier journals, Springer-Verlag Lecture Notes series (SVLN), Journal of Machine Learning Research (JMLR) and Machine Learning Journal (MLJ). The complexity of such a dataset is considerable, and concerns several aspects of the dataset: the journals layout styles are quite similar, so that it is not easy to grasp the difference between them; moreover, the 353 documents are described with a total of 67920 atoms, for an average of more than 192 atoms per description (some descriptions are made up of more than 400 atoms); last, the description is heavily based on a part_of relation that increases indeterminacy.

The $k$-NN approach performance was compared to that of a concept learning algorithm embedded in the learning system INTHELEX [5]. Specifically, two versions of such a system were exploited: the classical one (I) and a new one (SF) whose generalization operator was modified to take advantage from the pro-

\footnotetext{
${ }^{1}$ Available at http://lacam.di.uniba.it: 8000 /systems/inthelex/index.htm\#datasets
} 
posed similarity framework for identifying the best subdescriptions to be put in correspondence [8]. The performance was evaluated according to both Predictive Accuracy percentage and F-measure (with parameter 1 in order to equally weight Precision and Recall), to ensure that the performance was balanced between positive and negative examples. Classification figures for the concept learning algorithm, averaged on the 10 folds, are reported in Table 1: $C l$ is the number of clauses in the learned theories; Gen the number of generalizations carried out; $\mathrm{Spec}^{+}, \mathrm{Spec}^{-}$and $\mathrm{Exc}^{-}$ the number of specializations (by means of positive atoms, negated atoms and exceptions, respectively). The similarity-driven version outperformed the classical one in all considered parameters (time, learning behaviour, accuracy and F-measure); overall, in the 40 runs it saved 1.15 hours, resulting in a $98 \%$ average accuracy $(+1 \%$ with respect to the old version) and $96 \%$ average $\mathrm{F}$-measure $(+2 \%$ with respect to the old version).

For the $k$-NN approach, $k$ was set to the square root of the number of learning instances, i.e. 17. Notice that the classification was a multi-class one, so (although $k$ is odd) the nearest neighbours are not bound to a binary classification and ties are possible (indeed, 0.5 errors for SVLN in fold 8 means that two classes were the nearest ones, of which one correct). The results of the $k$-NN classification performance are summarized in Table 2, detailed for each fold. Some interesting considerations can be drawn upon these figures. First of all, the overall accuracy is $94.37 \%$, which means that few documents were associated to the wrong class, and hence the distance technique is very good in identifying the proper similarity features within the given descriptions. Actually, very often in the correct cases not just the majority, but almost all of the nearest neighbors to the description to be classified were from the same class. Then, note that classes Elsevier and MLJ always have 100\% accuracy, which means that the corresponding examples are quite significant and sharply distinguishable. Errors were concentrated in the SVLN and JMLR classes, where, however, high accuracy rates were reached. In detail, of the 13 JMLR wrongly classified observations, 8 were confused with Elsevier ones, and 5 as SVLN ones. Of the SVLN errors, 6 concerned Elsevier, 1 JMLR and 1 was a tie between the correct class and JMLR. This reveals that MLJ is quite distinct from the other classes, while Elsevier, although well-recognizable in itself, is somehow in between JMLR and SVLN, which are also close to each other. Interestingly, mismatchings concerning Elsevier are unidirectional: JMLR and SVLN are sometimes confused with Elsevier, but the opposite never happened; on the other hand, in the case of JMLR and SVLN it is bidirectional, suggesting a higher resemblance between the two.

As to the overall comparison to the concept-learning algorithm, it is very interesting to note that, while high performance on Elsevier and low performance on SVLN are confirmed from the conceptual learning case, for MLJ and JMLR the behavior of the two approaches is opposite, suggesting somehow complementary advantages of each. Elsevier confirmed full accuracy with respect to the similarity-guided version (the non-guided version had a slightly worse performance), while MLJ improved the performance up to full accuracy in the $k$ $\mathrm{NN}$ approach, gaining $4 \%$ over the guided version and $7 \%$ over the non-guided one. Conversely, accuracy on the remaining classes neatly decreased of about $-8 \%$. This can be explained with the fact that printed journals impose a stricter fulfillment of layout style and standards, and hence their instance are more similar to each other. Thus, the concept learning algorithm is more suitable to learn definitions that generalize on peculiar features of such classes.

Runtime refers almost completely to the computation of the similarity between all couples of observations: it takes an average of about 2sec computing each single similarity, which is a very reasonable time considering the descriptions complexity and the fact that the prototype has not been optimized in this preliminary version.

In order to check whether the novel similarity function was actually useful, or just the procedure for assessing the components similarity determined the performance, a comparison to other measures in the literature was made according to average precision, recall and accuracy for each dataset size, plus some information about runtime and number of description comparisons to be carried out. Results revealed an improvement with respect to both Jaccard's, Tverski's and Dice's measures up to $+5,48 \%$ for precision, up to $+8,05 \%$ for recall and up to $+2,83 \%$ for accuracy, confirming that also the basic function improved the state-of-the-art.

\section{Conclusions and Future Work}

The presence of relations in First-Order Logic causes the problem of indeterminacy in mapping portions of a description onto another one. Hence, the space induced by the descriptions is not Euclidean, and no straightforward way is available for assessing the similarity between two descriptions according to a distance measure. However, some real-world problems require the power of relations to be properly repre- 
Table 1. Classification results

\begin{tabular}{|c|c|c|c|c|c|c|c|c|c|}
\hline & & Time (sec.) & Cl & Gen & Spec $^{+}$ & Spec $^{-}$ & Exc $^{-}$ & Accuracy & F1-measure \\
\hline \multirow{2}{*}{ JMLR } & SF & 588.97 & 1.9 & 8.8 & 1.1 & 0 & 0.6 & 0.98 & 0.97 \\
& $\mathrm{I}$ & 1961.66 & 1.9 & 9.1 & 1.3 & 0.1 & 1.3 & 0.98 & 0.97 \\
\hline \multirow{2}{*}{ Elsevier } & $\mathrm{SF}$ & 52.92 & 1 & 6.3 & 0 & 0 & 0 & 1 & 1 \\
& $\mathrm{I}$ & 303.85 & 2.1 & 10.1 & 2.2 & 0.2 & 0 & 0.99 & 0.97 \\
\hline \multirow{2}{*}{ MLJ } & $\mathrm{SF}$ & 3213,48 & 4.7 & 15.7 & 3.7 & 2.5 & 0.8 & 0.96 & 0.94 \\
& $\mathrm{I}$ & 2974.87 & 5.2 & 14.5 & 4.4 & 3 & 2.1 & 0.93 & 0.91 \\
\hline \multirow{2}{*}{ SVLN } & $\mathrm{SF}$ & 399 & 2.6 & 8.1 & 0.7 & 0.4 & 1 & 0.98 & 0.94 \\
& $\mathrm{I}$ & 662.89 & 3.3 & 9.4 & 2.8 & 0.9 & 0.6 & 0.97 & 0.93 \\
\hline
\end{tabular}

Table 2. Results of the proposed approach

\begin{tabular}{|c||c|c|c|c|c|c|c|c|c|c||c|}
\hline Fold & 1 & 2 & 3 & 4 & 5 & 6 & 7 & 8 & 9 & 10 & \\
\hline \hline JMLR & 100 & 85.71 & 85.71 & 84.62 & 76.92 & 100 & 92.31 & 100 & 83.33 & 91.67 & Avg: 90.03 \\
errors & $0 / 14$ & $2 / 14$ & $2 / 14$ & $2 / 13$ & $3 / 13$ & $0 / 13$ & $1 / 13$ & $0 / 13$ & $2 / 12$ & $1 / 12$ & Tot: $13 / 131$ \\
\hline Elsevier & 100 & 100 & 100 & 100 & 100 & 100 & 100 & 100 & 100 & 100 & Avg: 100 \\
errors & $0 / 5$ & $0 / 6$ & $0 / 6$ & $0 / 5$ & $0 / 5$ & $0 / 5$ & $0 / 5$ & $0 / 5$ & $0 / 5$ & $0 / 5$ & Tot: $0 / 52$ \\
\hline MLJ & 100 & 100 & 100 & 100 & 100 & 100 & 100 & 100 & 100 & 100 & Avg: 100 \\
errors & $0 / 11$ & $0 / 11$ & $0 / 10$ & $0 / 9$ & $0 / 9$ & $0 / 9$ & $0 / 9$ & $0 / 10$ & $0 / 10$ & $0 / 12$ & Tot: $0 / 100$ \\
\hline SVLN & 83.33 & 100 & 100 & 100 & 100 & 62.5 & 87.5 & 92.86 & 87.5 & 83.33 & Avg: 89.70 \\
errors & $1 / 6$ & $0 / 5$ & $0 / 6$ & $0 / 8$ & $0 / 8$ & $3 / 8$ & $1 / 8$ & $0.5 / 7$ & $1 / 8$ & $1 / 6$ & Tot: $7.5 / 70$ \\
\hline \hline Overall & 97.22 & 96.43 & 94.29 & 94.29 & 91.43 & 91.43 & 94.29 & 98.57 & 91.43 & 94.29 & Avg: 94.37 \\
errors & $1 / 36$ & $2 / 36$ & $2 / 36$ & $2 / 35$ & $3 / 35$ & $3 / 35$ & $2 / 35$ & $0.5 / 35$ & $3 / 35$ & $2 / 35$ & Tot: $20.5 / 353$ \\
\hline
\end{tabular}

sented, and many approach in Artificial Intelligence in general, and in Machine Learning in particular, are based on the evaluation of similarity/distance between instances. This paper tackles the case of $k$-Nearest Neighbor classification, and proposes a novel similarity framework for FOL descriptions based only on their syntactic structure.

Based on the experimental outcomes of such a framework on the real-world task of document classification, it seems a viable solution that does not require deep knowledge of the domain and of the specific descriptors, still providing high performance on a difficult domain, comparable to those of a concept learning algorithm.

Future work will concern fine-tuning of the similarity computation methodology, and its application to other problems, such as flexible matching. Further experiments for $k$-Nearest Neighbour classification on other real-world datasets are currently undergoing, and other scheduled work includes coupling $k$-NN with clustering: clustering with this measure has already shown very good performance, comparable to that of supervised learning [8], and $k$-NN could be exploited to classify new instances into one or more of the induced clusters according to their full set of instances or just their prototypes. This would be particularly interesting in dynamic environments such as Digital Libraries management, where documents of unknown class in the repos- itory must be grouped into significant classes and then new incoming documents must be associated to the best groups among those available in the repository.

\section{References}

[1] G. Bisson. Learning in FOL with a similarity measure. In W.R. Swartout, editor, Proc. of AAAI-92, pages 82-87, 1992.

[2] S. Ceri, G. Gottlob, and L. Tanca. Logic Programming and Databases. Springer, 1990.

[3] P. Domingos. Rule induction and instance-based learning: a unified approach. In Proc. of IJCAI95, pages 1226-1232. Morgan Kaufmann, 1995.

[4] W. Emde and D. Wettschereck. Relational instance based learning. In L. Saitta, editor, Proc. of ICML-96, pages 122-130, 1996.

[5] F. Esposito, S. Ferilli, N. Fanizzi, T. Basile, and N. Di Mauro. Incremental multistrategy learning for document processing. Applied Artificial Intelligence Journal, 17(8/9):859-883, 2003.

[6] Floriana Esposito, Nicola Fanizzi, Stefano Ferilli, and Giovanni Semeraro. A generalization model based on oi-implication for ideal theory refinement. Fundam. Inform., 47(1-2):15-33, 2001. 
[7] S. Ferilli, T.M.A. Basile, N. Di Mauro, M. Biba, and F. Esposito. Similarity-guided clause generalization. In R. Basili and M.T. Pazienza, editors, AI*IA-200\%: Artificial Intelligence and Human-Oriented Computing, volume 4733 of Lecture Notes in Artificial Intelligence, pages 278289. Springer, Berlin, 2007.

[8] S. Ferilli, T.M.A. Basile, N. Di Mauro, M. Biba, and F. Esposito. Generalization-based similarity for conceptual clustering. In Z.W. Ras, S. Tsumoto, and D. Zighed, editors, MCD-2007, volume 4944 of Lecture Notes in Artificial Intelligence, pages 13-26. Springer, Berlin, 2008.

[9] Dekang Lin. An information-theoretic definition of similarity. In Proc. 15th International Conf. on Machine Learning, pages 296-304. Morgan Kaufmann, San Francisco, CA, 1998.

[10] J. W. Lloyd. Foundations of logic programming; (2nd extended ed.). Springer-Verlag New York, Inc., New York, NY, USA, 1987.

[11] C. Rouveirol. Extensions of inversion of resolution applied to theory completion. In Inductive Logic Programming, pages 64-90. Academic Press, 1992.

[12] S. Wieczorek, G. Bisson, and M. B. Gordon. Guiding the search in the no region of the phase transition problem with a partial subsumption test. In Machine Learning: ECML 2006, volume 4212 of LNCS, pages 817-824. Springer, 2006. 